\title{
PENG BLOCK AS AN ANALGESIC TOOL FOR TOTAL HIP ARTHROPLASTY A case series description
}

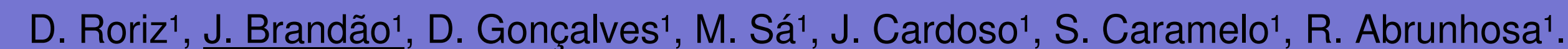

${ }^{1}$ Centro Hospitalar de Trás-os-Montes e Alto Douro, Anesthesiology and Pain Therapy, Vila Real (Portugal).

\section{Background}

Hip fracture is a common orthopedic emergency in the elderly, and is frequently associated with significant pain and morbidity. Popular analgesic regimens include peripheral nerve blocks such as femoral nerve block and fascia iliaca block. In this case series, we describe the analgesic efficacy and adverse effects of the recently described Pericapsular Nerve Group (PENG) block combined with Lateral Femoral Cutaneous nerve (LFC) block, in patients scheduled for urgent total hip arthroplasty (THA), as part of a multimodal pain strategy.

\section{Methods}

During the period from November, 2018 to April, 2019, 8 patients scheduled for THA underwent a single-injection block followed by spinal anesthesia. We performed a ultrasound-guided LFC $(5 \mathrm{ml})$ and PENG block $(15 \mathrm{ml})$ with $0.375 \%$ ropivacaine.

Post-operative analgesia included fixed paracetamol $1000 \mathrm{mg}$ IV (6h/6h) and tramadol $100 \mathrm{mg}$ IV (8/8h) with IV morphine as rescue analgesia.

At 24 hours post-operatively all patients were evaluated for analgesic efficacy, need for rescue analgesia, motor/sensory impairment of the thigh and common anesthetic complications and lateral effects.

\section{Results}

- No patient required rescue analgesia.

- Only two patients reported mild pain at rest.

- Two patients reported mild pain and three reported moderate pain with hip movement.

- Only one had paresthesia in a small area of the anterior thigh.

- No quadriceps weakness was clinically evident.

- No complications were noted.

Regional analgesia techniques are commonly used for pain management in patients with hip fractures. They provide reasonable analgesia with an opioid-sparing effect and are relatively safe.

Our results show that PENG block may be an overall safe and useful analgesic technique for hip surgery. Main advantages include a potential motor block-sparing effect and reduction in opioid-related adverse effects.

Although in agreement with current literature, further studies are required to address what is the ideal regional analgesia regimen for hip surgery.

\begin{tabular}{|c|c|c|c|c|c|c|c|c|c|c|c|c|c|}
\hline & \multicolumn{5}{|c|}{ Pain } & \multicolumn{2}{|c|}{$\begin{array}{c}\text { Rescue } \\
\text { Analgesia }\end{array}$} & \multicolumn{4}{|c|}{ Blockade } & \multicolumn{2}{|c|}{ Complication } \\
\hline & \multicolumn{2}{|c|}{ Rest } & \multicolumn{3}{|c|}{ Movement } & Yes & No & Ser & itive & Mo & & Yes & No \\
\hline & Ro & $\mathrm{R} 1$ & MO & M1 & M2 & \multirow{2}{*}{0} & \multirow{2}{*}{8} & S1 & $\mathrm{S} 2$ & M1 & M2 & \multirow{2}{*}{0} & \multirow{2}{*}{8} \\
\hline № & 6 & 2 & 3 & 2 & 3 & & & 7 & 1 & 8 & 0 & & \\
\hline
\end{tabular}

Table 1 Postoperative evaluation of analgesic efficacy.

Legend: R0, without pain; R1, mild pain; M0, without pain; M1, mild pain; M2, moderate pain; S1 without sensitive block; S2, sensitive block in small area; M1, without motor block; M2, motor block in small area. 